\title{
Perspectiva sociológica del contrabando de combustible en la frontera colombo - venezolana
}

Sociological perspective of the smuggling of fuel in the colombo-venezuelan border

\author{
Felipe González Palma ${ }^{1}$ \\ Universidad de Córdoba
}

Recibido el 27 de junio de 2017, aceptado el 27 de julio de 2017

Disponible en internet el 12 de agosto de 2017

\begin{abstract}
Resumen: este escrito contrasta la concepción clásica del derecho que ignora realidades para construir ficciones, como lo sostenía Marx respecto al hurto de leña, respecto de la acumulación por desposesión y bienes comunes, mostrando las dinámicas internas vinculadas a la práctica del contrabando de gasolina en la frontera colombo-venezolana. La metodología utilizada es descriptiva, desde un enfoque sociológico, en cuanto busca precisar cómo se manifiesta el fenómeno social objeto de estudio, intentando abordar sus principales características, en aras de lograr establecer una descripción del fenómeno social. Los hallazgos indican que el contrabando ilegal de combustibles se relaciona con la integración social del individuo en la sociedad fronteriza, que hacen plausible su comprensión desde una perspectiva cultural, entendida esta como constructo eminentemente social, lo cual desborda la lectura determinista que señala que la problemática social del contrabando de combustible obedece simple y llanamente a factores de orden económico o bien al abandono por parte del Estado.
\end{abstract}

Palabras claves. Sociología, contrabando, desposesión de bienes, derecho

\section{INTRODUCCIÓN}

La realidad como construcción socialmente producida (Berger/Luckmann, 1966) implica una relación dialéctica entre Cultura y Naturaleza, relación que se encuentra indisolublemente unida, estando el hombre entonces como organismo biológico y como ser social atado a esa dialéctica insalvable. Bajo esas premisas, lo que llamamos «orden social» es una producción fundamentalmente humana de carácter constante en la cual es el ser humano a través de un proceso complejo el que le da forma social a la naturaleza mediante la externalización, la objetivación y la internalización.

Cualquier intento de aproximación sociológico a un fenómeno social debe entonces partir de la premisa fundamental de que la sociedad es una producción constante en el que

\footnotetext{
${ }^{1}$ Abogado, Especialista en Derecho Público. Candidato a Magister en Derecho Público. Correo: felipedgp81@gmail.com
} 
indistintamente se realizan los tres momentos de ese proceso dialectico anteriormente señalados. En la sociedad -al igual que en un organismo biológico- se presentan problemas, que en realidad constituyen oportunidades, a nivel biológico los problemas producen adquisiciones evolutivas que ayudan a resolverlos, y a nivel social dichas adquisiciones evolutivas se manifiestan en instituciones que surgen de dichos problemas sociales. Por ello, el estudio de las problemáticas sociales encuentra una importancia cardinal.

Podemos establecer claramente que en Colombia son múltiples las problemáticas sociales que, valga la redundancia, azotan a la sociedad, muchas de ellas se acentúan y van variando a medida que se pasa de una región a la otra, pero existe una en específico que pese a poder llamar mucho la atención al investigador social por la complejidad que reviste, no ha sido estudiada a fondo con la exigüidad y rigurosidad que amerita la misma y pocas veces recurriéndose a un análisis sociológico, nos referimos específicamente a la problemática social existente en la región de frontera entre Colombia y Venezuela consistente en el contrabando de combustible. En efecto, se trata de una problemática que presenta amplios matices, a los cuales intentaremos aproximarnos en este ensayo, a partir de presupuestos teóricos dados por la sociología, que nos permitan dar cuenta sobre la posible función social que cumple esta problemática en la región fronteriza, así como intentar aproximarnos a si la misma puede o no constituir una anomia social.

En ese orden de ideas, planteamos de antemano que el contrabando ilegal de combustibles en la frontera Colombo - venezolana se relaciona con la integración social del individuo en la sociedad fronteriza, que hacen plausible su comprensión desde una perspectiva cultural, entendida esta como constructo eminentemente social. Con este trabajo se busca que se entre a considerar a fondo el contrabando de combustible en la frontera como una verdadera problemática social, con lo que se busca aportar algunos elementos teóricos, alejados del clásico determinismo que señala que la problemática social del contrabando de combustible obedece simple y llanamente a factores de orden económico o bien al abandono por parte del Estado.

\section{Metodología}

La metodología que utilizaremos para abordar la problemática es descriptiva en cuanto buscaremos precisar cómo se manifiesta el fenómeno social objeto de estudio, intentando 
abordar sus principales características, en aras de lograr establecer una descripción del fenómeno social.

Así mismo, por las características propias de este ensayo, utilizaremos además una metodología de tipo cualitativa, por ello nos centraremos en el análisis de distintos textos de prensa digital, al igual que en diferentes investigaciones que han abordado la problemática del contrabando en general, y del contrabando de combustible en particular. Si bien un estudio de tipo cuantitativo permitiría arrojar más luces sobre la problemática social, lo cierto es que por las mismas dificultades que la misma reviste, no existe un censo o cifras confiables que permitan dar cuenta con absoluta certeza sobre aspectos fundamentales de la misma tales como el número de personas que se dedican a dicha actividad, o incluso las familias que dependen directa o indirectamente de ella.

El método cualitativo nos permitirá a través de un proceso inductivo en la relación teoríaconcepto y fenómeno social investigado dar cuenta sobre los aspectos esenciales de la problemática desde un enfoque sociológico, y teniendo como norte que la imagen de la realidad investigada es social.

\section{CUESTIONES CONCEPTUALES REFERENTES A LA PROBLEMÁTICA SOCIAL}

Resulta esencial para los propósitos de este ensayo, realizar unas precisiones conceptuales previas, referentes a la problemática social que pretendemos analizar. Si bien es cierto, que muchas veces las definiciones conceptuales producen un determinismo respecto a la problemática social, en este caso resulta fundamental, en cuanto existen distintos entendimientos acerca de la problemática social. En efecto, si se analizan los distintos trabajos académicos que han intentado realizar una aproximación a la problemática del contrabando de combustible, son muchas las nociones que la misma puede abordar. Una primera noción es la que identifica el contrabando de gasolina con los denominados pimpineros (Adarve, 2009). En esta definición pimpineros son las personas que se dedican a traer gasolina de contrabando de Venezuela para comercializarla en Colombia (Adarve, 2009, p. 167), se les dice pimpineros porque la etimología de la palabra viene del recipiente de cinco galones (al cual le llaman pimpina) en el que cargan el combustible.

Por otra parte; para Egea y Soledad (2013), pimpineros son las personas que se dedican a comercializar en las calles colombianas la gasolina traída de Venezuela, en esta noción el 
contrabandista de gasolina es reducido al mero vendedor ambulante de gasolina de contrabando en la frontera.

Nosotros utilizaremos la acepción más amplia posible de contrabandistas de gasolina, la cual asimilaremos en ese sentido amplio a los pimpineros, entendiéndola como toda persona y/o familia dedicada a la importación en forma ilegal de combustible de Venezuela, mediante procedimientos más o menos complejos, y a su posterior comercialización en territorio Colombiano. Bajo ello, en adelante podremos utilizar en forma indistinta el termino "contrabandista de combustible" con el de "pimpineros", lo cual se repite, para los efectos de este trabajo, serán asimilados, por lo que diremos que efectivamente de lo que se trata en ultimas es de un rol social. Y precisamos un concepto ceñido a la delimitación espacial a la frontera colombo venezolana, pues si bien es cierto el fenómeno de contrabando de combustibles es una problemática que se puede presentar en los más de 6000 kilómetros cuadrados de fronteras que comparte Colombia con distintos países ${ }^{2}$, es en la frontera en mención donde reviste características culturales y simbólicas propias que permiten un mejor análisis de la problemática social, haciendo especial énfasis en los departamentos de Norte de Santander, Santander y la Guajira. En esta definición apartamos la práctica de contrabando ilegal de combustibles realizadas por estructuras criminales como paramilitares o bandas criminales.

Esto nos permite dar cuentas de varios elementos en la cadena de esta problemática social, el primer elemento en la cadena es quién compra el combustible en territorio venezolano, el segundo elemento es la introducción de ese combustible a territorio colombiano (en esta etapa es donde se utilizan procedimientos más o menos complejos para llevar a cabo ese cometido), y el último elemento es la comercialización del mismo, generalmente realizada en la misma zona fronteriza, al aire libre y a la vista de todos (Adarve, 2009, p.168).

Ahora bien, en lo recorrido en este escrito hasta el momento hemos dado por sentado de que en efecto el contrabando de combustibles en la frontera colombo venezolana es una problemática social, pero ¿ello es realmente así desde un punto de vista sociológico?

\footnotetext{
${ }^{2}$ El contrabando de combustible es una problemática social que se presenta también en la frontera de Colombia con Ecuador, país en el que también existen subsidios a los combustibles, lo que hace atractivo su importación ilegal a territorio Colombiano. Si bien es cierto, al igual que la frontera Colombo venezolana, en la frontera Colombo Ecuatoriana no existen cifras confiables sobre la misma, según estimaciones realizadas el contrabando del gas licuado de petróleo llega al 22\%, de diésel al 13\% y de gasolina al 7\% (El Comercio, 2013), todo lo cual supone una problemática social de gran impacto.
} 
El sociólogo norteamericano Herbert Blumer (perteneciente a la corriente del interaccionismo simbólico) nos ofrece mayores luces que nos permiten determinar que efectivamente el contrabando de gasolina en la frontera colombo venezolana constituye un problema social. Blumer (1971) planteaba que "un problema social existe principalmente en los términos en que es definido y concebido en la sociedad"'( p.300), lo cual conlleva que (1) El problema emerge (2) El problema es legitimado (3) Existencia de una movilización social respecto al problema (4) Formulación de un plan oficial de acción respecto al problema y (5) Implementación del plan oficial de acción respecto al problema; lo que en ultimas indica que son los mismos actores sociales implicados lo que determinan la existencia o no del problema social.

Bajo ese modelo referente, es claro que ha sido la misma sociedad colombiana y venezolana (fronteriza o no fronteriza) la que ha determinado que el contrabando de combustible constituye en efecto un problema social, pues son muchas las familias que utilizan esta como medio para la subsistencia y su utilización desde un punto de vista objetivo, implica la infracción a la ley, así como al orden económico (1); además por su múltiple presencia y tratamiento en medios y en instituciones públicas el problema se ha sido legitimado (2) constituyéndose en un punto en el que interactúan diferentes intereses, intenciones y fines, dándose por tanto una movilización social de la sociedad para que esta problemática social sea enfrentada (3), con especial interés de la sociedad fronteriza, por lo que existe un plan oficial de las instituciones de carácter público a fin de formular distintas acciones respecto al mismo (4) tales como soluciones económicas y sociales a los pimpineros (ver Caracol Radio, 2016) y su legalización (ver Rcn Radio, 2016)), todo lo cual se ha ido implementado (ver El Tiempo, 2011) (5), planes de acción que dan cuenta simbólicamente de la existencia de la problemática social (ver Cúcuta 7 Días, 2016).

Teniendo entonces meridianamente claro que el contrabando de combustibles en la frontera colombo venezolana constituye una problemática social en cuanto la sociedad ha construido una representación en la cual se le ha hecho la consideración como tal, resulta entonces pertinente en la siguiente parte de este ensayo, entrar a describir la problemática social. 


\section{DESCRIPCIÓN DE LA PROBLEMÁTICA SOCIAL}

En términos generales la problemática del contrabando de combustibles en la frontera colombo venezolana hace parte de una problemática mucho más amplia constituida esencialmente por el contrabando. Como ello es así, para determinar si existe o no existe un proceso complejo de externalización-objetivación-internalización en la sociedad fronteriza respecto a la problemática del contrabando en general y la del contrabando de combustibles en específico, conviene realizar un breve recorrido histórico acerca de la misma, que nos permita en un análisis concreto determinar cómo han operado ellas, teniendo presente que las mismas si bien son momentos de un proceso, no ocurren como si se tratase de una secuencia.

La frontera Colombo Venezolana está compuesta espacialmente por cerca de 2.219 kilómetros, y por tradiciones ancestrales de intercambio entre uno y otro lado. Es por ello, que históricamente, desde que se crearon las fronteras artificiales esquematizadas en Estados Nacionales que llamamos "Colombia" y "Venezuela", ha existido un fuerte componente de intercambio comercial y cultural entre ambos países, que ha creado referentes culturales propios de las regiones de frontera.

En ese marco, lejos de las valoraciones que puedan realizarse respecto a categorías de "bueno" o "malo", lo cierto es que el contrabando es un referente cultural de la región de frontera, siendo incluso utilizado como tal en distintas expresiones y tradiciones culturales, que podemos fácilmente encontrar con la escucha de diversos temas musicales autóctonos de la región de frontera (ver entre otros, las composiciones musicales "El contrabandista" de Sergio Moya Molina, "El Almirante Padilla” de Rafael Escalona).

Como resulta natural, la construcción de ese referente cultural, se remonta a la época colonial, especialmente durante el siglo XVIII donde según (González, 2008, p. 26-27) el contrabando se había constituido en una actividad ampliamente generalizada en el territorio de lo que hoy denominamos Colombia, siendo especialmente relevante en el territorio fronterizo que hoy llamamos "La Guajira" donde se concentraba el mayor paso de contrabando, el cual se encontraba constituido principalmente por mercancías tales como cueros, palos de Brasil, tabaco y ganado. 
Tal como puede presentarse obvio para el lector, lo cierto es que anterior a los procesos de colonización, no es posible encontrar referencias en pueblos indígenas a categorías similares de lo que podemos entender como "contrabando", siendo este un patrón totalmente ajeno a las organizaciones sociales que habitaban en la época pre-colonial en territorio colombiano. De tal forma, que es con la colonización que se introduce el contrabando, con la existencia de los primeros asentamientos, contrabando, que dicho sea de paso, fue una categoría introducida por piratas y mercaderes europeos, siendo entonces una categoría socialmente construida en Europa, y traída con la colonización al «nuevo mundo».

Ahora bien, el recorrido histórico hecho por (González, 2008) permite dar cuenta que si bien se trata de una categoría introducida por los europeos, lo cierto es que la misma ha sido practicada por indígenas, blancos, negros, mestizos y extranjeros, lo que en cierta forma evidencia que esto ha sido posible a través de un proceso de externalizaciónobjetivación-internalización, siendo especialmente relevante en los pueblos indígenas, de la que el pueblo Wayuu es digno ejemplo. Matute Campusano (2003) expresa esa peculiaridad de los pueblos indígenas en los siguientes términos "el guajiro y el Wayúu se refieren al contrabando como un comercio tradicional y consideran tener un derecho propio sobre el mismo, motivo por el cual no conciben la palabra ilegalidad para aludir a él" (p. 46), lo cual da muestra de la forma en como se ha apropiado a través del mencionado proceso(externalización-objetivación-internalización) de categorías que prima facie son ajenas a los pueblos indígenas, pero que toman en la dinámica social de esos pueblos concretos el carácter de actividad humana objetivada, lo cual refleja además la falta de necesidad de una coherencia a priori entre instituciones que hiciera posible la transmisión de significados entre una sociedad "civilizada" y una sociedad de "cazadores" o "recolectores".

Agrega Campusano (2003) que "el poder del contrabando parece radicar en el argumento de la costumbre y en su transcurrir histórico, lo que conlleva a una ancestralidad y territorialidad" (p. 73), con lo que se puede decir que respecto al contrabando ha ocurrido una verdadera "sedimentación" en los términos propuestos por Berger y Luckmann (1966, p.91), en lo cual inevitablemente las experiencias respecto a la misma quedan estereotipadas en el recuerdo como entidades memorables y reconocibles, en este caso en forma intersubjetiva, siendo verdaderamente social por haberse objetivado en experiencias 
compartidas, y dándose su significación a partir de su ancestralidad que no es otra cosa que la tradición reflejada en la trasmisión de una generación a otra. Bajo ese presupuesto, alrededor del contrabando y en referencia al mismo se han creado un orden simbólico reflejado en creencias, valores y sentimientos.

Ese orden simbólico reflejado en creencias, valores y sentimientos tiene implicación directa en la socialización, que de acuerdo a la sociología, "es el proceso mediante el cual los niños y otros nuevos miembros de la sociedad aprenden la forma de vida de esta" (Giddens, 2001, p. 58). Bajo ese entendido es posible afirmar que respecto al contrabando existen procesos fundamentales de socialización, pues es evidente que la misma como patrón cultural de la sociedad fronteriza incide en la formación que tendrán los nuevos miembros de dicha sociedad. Y decimos "incide" de forma intencional, en la medida en que dicha socialización, de acuerdo a las enseñanzas de (Giddens, 2001, p. 59), no es un tipo de "programación cultural" en la cual el niño absorbe en forma automática e inevitable las influencias de su contorno social, de tal manera que los individuos de la sociedad fronteriza serán inexorablemente "contrabandistas", pues no se trata de un proceso meramente pasivo, sino que el individuo a partir de su conciencia también entra a determinar y a incidir socialmente, ya que lo que en el fondo se trata es de la determinación social de conciencias individuales, las que a su vez, en un proceso continuo, afectan a su vez su contorno social desde el mismo momento de su nacimiento.

Cuando el individuo fronterizo internaliza la sociedad en la socialización primaria, el patrón cultural del contrabando indudablemente es una de las formas sociales con las cuales se ve "familiarizado" en su crecimiento. Por ello, no es casualidad que muchas de las personas que se dedican al contrabando (en especial el de combustible, como da cuenta Adarve, 2009, p. 167) lo hagan en familia, y en esa medida no resulta ingenuo, siendo conveniente recordar que la familia es el principal agente socializador durante la fase de la socialización primaria (Giddens, 2001, p.59). 
Estas consideraciones realizadas respecto al contrabando en "general", se pueden extender al contrabando de combustible, si bien es cierto su aparición se remonta a épocas menos pretéritas $\left(1980^{3}\right)$, los elementos de una y otra son los mismos, salvo pequeñas variaciones.

Pero continuando con los procesos de socialización, lo cierto es que es la socialización secundaria las que nos brinda muchos elementos de análisis que nos permiten establecer la manera como el contrabandista de combustible constituye y se construye como rol social en la sociedad fronteriza. La socialización secundaria, al igual que la primaria, constituye un proceso, pero en esta el individuo se ve inducido hacia sectores nuevos del "mundo" de su sociedad, siendo especialmente relevante en dicho proceso la adquisición de conocimientos específicos de roles.

Siguiendo con ello, y teniendo como respaldos las teorías de Merton y Berger/Luckmann es posible establecer que el "pimpinero" constituye en efecto un rol social, si se analiza la generalidad de los mismos, en los cuales la mayoría tienen el contrabando de combustible como un negocio familiar, se puede discernir que este tipo de personas no se vuelven pimpineros de la noche a la mañana, sino que es necesario que los mismos pasen a través de una serie de fases donde sea posible el establecimiento del hábito de contrabandear con combustible, sobre todo y en mayor medida porque la práctica del mismo requiere de una serie de utilización de métodos y/o herramientas susceptibles de aprehender a través -valga la redundancia- del aprendizaje mediante repetición o habituación. Los agentes de socialización, incluyendo a la familia, tienen una fuerza socializadora que incide en el individuo y que conlleva hacia la configuración del rol social.

Bajo esos parámetros, esta categoría que hemos querido llamar para estos efectos como "rol social de pimpinero", hace posible una reformulación de la problemática social. Al crecer al interior de un grupo social se aprende a considerar los patrones o comportamientos que se dan en el mismo, creándose el criterio de que los mismos pueden aceptarse dentro de categorías como "normales" o "anormales". En el contexto fronterizo el rol social del pimpinero se considera dentro de una categoría de lo que es posible considerar como "normal". A entender la problemática en esa manera, es posible discernir que la misma no

\footnotetext{
${ }^{3}$ De acuerdo con Egea y Soledad (2013, pp. 94-95), es posible ubicar el origen del contrabando de combustible a partir de la década de 1980, cuando diversas situaciones de carácter económico favorecieron la inusual práctica para el momento.
} 
se produce como consecuencia necesaria de la posible "falta de Estado" o por la falta de sanciones por parte del Derecho, o bien porque supone una determinación lógica necesaria dada por factores económicos atinentes principalmente a la motivación que puede presentar las diferencias dadas por las divisa colombiana y venezolana. Con esto no queremos dar al traste de entrada a esas posibles hipótesis que han querido abordar la problemática, si bien estas son hipótesis que se presentan como plausibles y que por tanto, no deben ser descartadas de plano, sino entenderlas en una forma integradora con la visión que aquí se intenta proponer de la misma como constitutiva de roles sociales, con las implicaciones que ello tiene.

Por supuesto que la diferencia de poder adquisitivo entre la moneda colombiana y venezolana (Mojica/Paredes, 2004, pág. 20), que ha sido una tendencia histórica, así como los subsidios con que se vende en Venezuela el combustible, constituyen motivaciones innegables que conllevan a la acentuación o no acentuación de la problemática social tratada en este pequeño ensayo, pero en ese mismo sentido, es igualmente innegable que en el análisis de los distintos roles sociales resulta imprescindible que para los mismos se presenten motivaciones que se deben entender, en nuestro criterio, como referentes emotivos del rol social, que igualmente va acompañado de otros referentes de carácter simbólico, tradicional y valorativo.

Bajo ese orden ideas, la diferencia de poder adquisitivo de la moneda constituye solo un referente de carácter emotivo o motivacional del rol social del pimpinero, que se presenta durante el continuo y dialectico proceso de socialización, siendo más evidente en la socialización secundaria, pues el individuo a lograr identificar con mayor facilidad basarse en las instituciones, se le permite discernir con claridad el referente motivacional en mención, con lo que se demuestra que es importante reconocer como el proceso de socialización permite entender los distintos patrones culturales y de conducta.

En ese mismo sentido, la "pimpina" no puede ser vista como un simple objeto, sino como un verdadero referente simbólico en la zona fronteriza. Frente a la misma ha operado un proceso que ha conllevado hacia la internalización de ella como referente. Todo esto, a su vez, junto a los procesos de socialización y los distintos procesos históricos ha representado el hecho de que se haya producido una legitimación implícita del contrabando de combustible. 
Por su parte, el combustible ha adquirido un carácter misterioso, el mismo se ha aprehendido como una realidad no humana, o en mejores términos, como si se tratase de una entidad onticamente dada, que se asimila a su vez al concepto de "reificación", por supuesto esto resulta evidente en cuanto el combustible constituye una mercancía, y de acuerdo a Marx (1867, p. 37) frente a ellas opera un fenómeno en virtud del cual se proyecta el denominado "carácter social" del trabajo del ser humano como si se tratase de un carácter material propio de los productos dados por el trabajo humano.

Es por ello, que el combustible es uno de los objetos sociales más importantes de la sociedad fronteriza, su importancia es tal que lleva a muchos individuos a asumir el rol social de pimpinero, sabiendo conscientemente que la misma supone un alto riesgo para su vida, por la volatilidad e inflamación inmanente a este producto, de tal forma que el producto es más importante incluso que la misma vida del individuo. El contrabando de combustible, ofrece serios riesgos para la vida de las personas que se dedican a la misma, los poco ortodoxos métodos utilizados para su transporte de territorio venezolano a territorio colombiano, los cuales se encuentran desligados de medidas de seguridad mínimas, así como las formas en la que se comercializa en territorio colombiano (generalmente al aire libre en lugares no provistos de medidas para tal fin), suponen un serio peligro, el cual se encuentra latente en todas las etapas de la cadena que supone el contrabando de gasolina.

\subsection{BANDAS CRIMINALES Y EL CONTRABANDO DE COMBUSTIBLE: ANOMIA Y ESTRUCTURA} SOCIAL

En el contrabando de combustible no solo participan personas de la fronteras individualmente consideradas, también actúan estructuras criminales que se han apoderado de muchos de los eslabones de contrabando de combustibles. Dichas estructuras criminales, en un primer momento fueron paramilitares (Adarve, 2009, p.171) y hoy resultan ser las mal llamadas "bandas criminales" (ver El Tiempo, 2014, Colombia Informa 2013, El Pilón 2013). Si bien habíamos dicho que respecto al llamado "pimpinero" se trataba de un rol social, mal podríamos extender esa consideración hacia el contrabando de combustible realizado por estas estructuras criminales, pues en el primer caso se trata de una práctica ancestral y en el segundo caso no se trata de nada de eso. Esto punto en concreto, nos pone 
de frente para plantear si en este evento, el contrabando de combustible se trata de una posible conducta desviada, con las implicaciones que ella trae.

El análisis del contrabando de combustible como posible conducta desviada nos debe llevar necesariamente hacia el estudio de los conceptos de anomia y estructura social. El primer concepto, es propuesto por Emile Durkheim (1883, p. 433), el mismo hace referencia a una falta relativa de normas de una sociedad o grupo. A partir de la modernidad, la división del trabajo produce una desintegración en la sociedad, que se ve reflejada en una especialización de la misma; dicha división del trabajo -que constituye un elemento esencial de la modernidad al igual que la especialización- no es una necesidad de la naturaleza, es una creación social producida por las transformaciones sociales. La división del trabajo y la especialización generan diversidad en la sociedad, y para que en esa diversidad se establezca lazos solidos es necesaria la reglamentación que regule las nuevas relaciones sociales que surgen en la sociedad, de lo contrario, la ausencia de esa reglamentación debilita la moral pública, lo que se identifica con la anomia, que resulta representar un peligro en cuanto los individuos empiezan a dejar de tener claridad entre lo que es justo y lo injusto, al igual que lo legitimo y lo ilegitimo (Durkheim 1883, p.13-17).

El concepto de anomia, se encuentra a su vez ligado al concepto de estructura social desarrollado por Merton en Teoría y Estructuras Sociales (1964), para este autor la anomia se concibe como el quiebre de un estructura cultural por lo que "hay una separación aguda entre las normas y los objetivos culturales y las capacidades socialmente estructuradas de los individuos del grupo para obrar de acuerdo con aquellos." (p. 170).

Un análisis del contrabando de combustible realizado por estas estructuras criminales en el contexto fronterizo, permite identificar algunos elementos que hacen pensar que la misma se trata, en efecto, de una anomia. Por una parte, la práctica en este contexto significa una separación entre las normas y los objetivos culturales con los que históricamente se ha justificado el contrabando al interior del grupo social, lo cual se corrobora con lo expresado por (González, 2008) en el análisis histórico que realiza de la problemática del contrabando en los siguientes términos: "el problema central que plantea La Guajira es que la existencia de una práctica como el contrabando tradicional de mercancías producidas legalmente, legítima para muchos a partir de unas circunstancias socioculturales e históricas 
determinadas, sentó las bases para el desarrollo posterior de prácticas delincuenciales y criminales." (p.18).

En ese mismo sentido, es claro que la relación Centro-Periferia, que ubica a la zona fronteriza en la periferia, evidencia claramente que frente a las relaciones sociales que en la misma se presenta, existe cierto margen de penumbra en cuanto a las nociones de legalidad/ilegalidad y legitimo/ilegitimo, no siendo coincidentes en muchos casos ambos, y siendo pertinente resaltar que uno de los aspectos que ha posibilitado la extensión histórica del contrabando es precisamente esa falta de claridad entre lo legitimo y lo ilegitimo. Y por otra parte, la misma representa un desorden social en el contexto fronterizo.

Siguiendo con Merton (1964) nos dice que "todas las formas sociales o culturales estandarizadas tienen funciones positivas" (p. 41), pero en el mismo sentido, y que constituye uno de los grandes aportes del autor, estas formas sociales o culturales a su vez presentan también funciones negativas o desintegradoras. Esto nos lleva a considerar para el caso concreto del contrabando de combustible que efectivamente en la misma se presenta lo que Merton denomina funciones manifiestas y funciones latentes. En nuestro criterio, se presenta como claro que en el evento del rol social del pimpinero, nos encontramos en presencia de una función manifiesta, pues se trata de una consecuencia necesaria que se presenta por la adaptación del sistema y que es querida y aceptada por los individuos, la cual consideramos ligadas a las expectativas generadas y esperadas por el rol mismo, que producen una función social integradora; y en el caso del contrabando ilegal de combustibles por parte de estructuras criminales como paramilitares y bandas criminales, nos encontramos en presencia de una función latente, pues se trata de una consecuencia no querida ni esperada (Merton, 1964, p. 139).

En ese marco, en nuestro criterio el contrabando de combustible puede verse analizado desde las orbitas teóricas de función manifiesta y latente.

\section{DEl hURTO DE LEÑA EN MARX AL CONTRABANDO DE COMBUSTIBLE}

Siguiendo el postulado de Marx, que señalaba al Derecho como una ideología, respecto a la problemática social que analizamos en este trabajo, podemos realizar consideraciones paralelas a las realizadas por el autor prusiano frente al hurto o robo de leña (Marx, 2007). 
Si bien las consideraciones hechas por Marx respecto a la penalización del llamado hurto de leña, van dirigidas a presentar una crítica mordaz contra el capitalismo y de la forma como el Derecho resulta ser una superestructura dependiente del orden económico, que se convierte en una vía idónea para la desposesión de bienes comunes, en nuestro criterio el asunto se trata en el fondo en como el Derecho se vale de artificios o ficciones jurídicas representadas en forma de leyes para penalizar practicas consuetudinarias. Dichas prácticas consuetudinarias -como es el caso del contrabando de combustible- significan el enfrentamiento de un interés público de un grupo o clase social contra intereses privados, que son en últimas los que se terminan imponiendo a través del Derecho y específicamente de las leyes.

El contrabando en general, que como hemos visto, se trata de una práctica consuetudinaria, una tradición ancestral en el contexto social de la frontera, solo fue catalogada por el Derecho como delito en el año 2000 a partir de la ley 599 (Código Penal), el cual ha sido modificado posteriormente por otras leyes.

Así mismo, el contrabando de combustible ha sido tipificado como delito a partir de la ley 788 de 2002, subsumiéndola en el tipo penal denominado "contrabando de hidrocarburos y sus derivados".

En esa forma, el contrabando de combustible, como practica consuetudinaria, ha sido encerrado -al igual que el hurto de leña- como delitos, mediante el proceso en el cual el Derecho se determina por las estructuras económicas, creando ficciones que ignoran realidades.

\section{A MANERA DE CONCLUSIÓN}

En el presente trabajo podemos llegar a las siguientes conclusiones respecto a la problemática que se tomó como referencia:

El contrabando en general se constituye como un referente cultural de la región de frontera, ha sido una práctica consuetudinaria de los habitantes de dicha región. Como categoría el contrabando es traída e introducida por los europeos, no necesitó de una "coherencia a priori" para ser transmitida a pueblos nativos, que la adoptaron a través de un proceso de externalización-objetivación-internalización. 
La socialización primaria y la socialización secundaria juegan a su vez un papel primordial para la construcción alrededor del contrabando, de roles sociales. En esa forma gracias a esos procesos, es posible identificar el rol social del pimpinero, como rol social tiene una función social positiva -en términos de Merton-, pues como rol produce la integración social de los individuos, bajo ello se puede identificar a la "pimpina" como un referente cultural. A su vez, siguiendo al mismo Merton, es posible determinar que respecto al contrabando de combustible, el rol social del pimpinero y la integración social que produce puede llegar a considerarse como una función manifiesta, mientras que respecto al contrabando de combustible por parte de bandas criminales y paramilitares, nos encontramos en presencia de una función latente, la cual constituye una verdadera problemática social por todos los factores que involucra la misma.

Respecto a la gasolina, como toda mercancía se ha producido una reificación que ha llevado al individuo a realizar la práctica social de contrabando de combustible, incluso a sabiendas de que la manipulación de la misma supone serios peligros para su vida.

El contrabando en general y el contrabando de combustible en particular, como práctica consuetudinaria, ha sido objeto de embates por parte del Derecho, que ignora realidades para construir ficciones, al igual que lo que sostenía Marx respecto al hurto de leña.

Referencias bibliográficas

Adarve, I. (2009). “LOS PIMPINEROS”. En Villegas M. \& Villegas M. (Autores), Normas de papel: La cultura del incumplimiento de reglas (pp. 167-176). Siglo del Hombre Editores.

Berger, P./Luckmann, Th. (1966). “The social construction of reality”. New York: Doubleday; (trad. al cast. de Silvia Zuleta), La construcción social de la realidad, Buenos Aires: Amorrortu 1968.

Blumer, H. (1971), “Social Problems as collective behaviour”, Social Problems, Volumen 18, Número 3, 1971, pp. 298-306

Campusano, M. (2003. "De matutes, corsarios y bajales... ¡a pesar del Estado, La Guajira vive!" (Trabajo de grado), Medellín, Universidad de Antioquia, carrera de Antropóloga. 
Colombia Informa (2013), "Pimpineros de Cúcuta acosados por paramilitares y administración municipal", 29 de Diciembre. Disponible en http://www.colombiainforma.info/pimpineros-de-cucuta-acosados-porparamilitares-y-administracion-municipal/

Durkheim, È. (1893). “De la división du travail social". Paris: Huitième; (trad. al cast. de Carlos García Posada), La división del trabajo social. Madrid: Akal 2001.

Egea, C., \& Soledad, I. (2013). "La venta informal de combustible en la frontera ColombiaVenezuela: El papel de los pimpineros como grupo vulnerable”. Revista de Ciencias Sociales (Ve), XIX(1), pp. 92-105.

El Pilón (2013), “A 'bacrim' atribuyen asesinato de'pimpineros' en Aguachica”, 6 de mayo. Disponible en http://elpilon.com.co/a-bacrim-atribuyen-asesinatodepimpineros-en-valledupar/

El Comercio (2013), "Contrabando de combustibles", 6 de Noviembre. Disponible en http://www.elcomercio.com/opinion/contrabando-combustibles.html.

El Tiempo (2011), "Pimpineros pasan a ser distribuidores", 5 de abril. Disponible en http://www.eltiempo.com/archivo/documento/MAM-4486704

El Tiempo (2014), "Bandas criminales están ahora tras negocio del contrabando" 31 de Julio. Disponible en http://www.eltiempo.com/colombia/otras-ciudades/bandascriminales-tras-negocio-del-contrabando/14329339

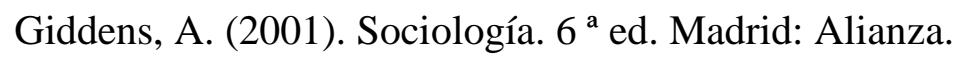

González, S. (2008). "Pasado y presente del contrabando en La Guajira aproximaciones al fenómeno de ilegalidad en la región”. Bogotá, Editorial Universidad del Rosario. Retrieved from http://www.urosario.edu.co/economia/documentos/BI_CEODD_10/

Marx, K. (1867).” Das Kapital. Kritik der politischen Ökonomie. Erster Band, Buch I: Der Produktionsprozess des Kapitals". $8^{\text {a }}$ ed. (1 $1^{\text {a }}$ ed. 1867, Otto Meissner - Hamburg), Berlin: Dietz; (trad. al cast. de Wenceslao Roces), El capital. Crítica de la economía política. Tomo I. $3^{\mathrm{a}}$ ed. (1946 $1^{\mathrm{a}}$ ed.). $4^{\mathrm{a}}$ reimpresión. México: Fondo de cultura económica 2008. 
Derecho \& Sociedad / Montería - Colombia

ISSN 2539-4401, p.p. 53-69

$\mathrm{N}^{\circ} 2 \mid$ VOL 1. AÑO 2017

Artículo de reflexión

Marx, K. (2007). “Los debates de la Dieta Renana”. Barcelona: Gedisa.

Merton, R. (1964), “Teoría y Estructuras Sociales”. México: Fondo de Cultura Económica.

Mojica, A. \& Paredes, J. (2004). "La economía Colombo - Venezolana y su impacto en la región de frontera. 1999 - 2003”. Bogotá: Banco de la Republica y Centro Regional de Estudios Económicos de Bucaramanga, 2004.

RCN Radio (2016), "Pimpineros de Norte de Santander buscan salir de la informalidad", 19 de agosto. Disponible en http://www.rcnradio.com/locales/pimpineros-nortesantander-buscan-salir-la-informalidad/ 